\title{
STAR FORMATION IN THE EARLY UNIVERSE: BEYOND THE TIP OF THE ICEBERG
}

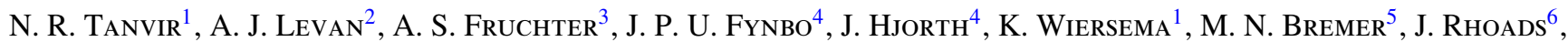 \\ P. Jakobsson $^{7}$, P. T. O’Brien ${ }^{1}$, E. R. Stanway ${ }^{2}$, D. Bersier ${ }^{8}$, P. NATARajan $^{9}{ }^{10}$, J. Greiner $^{11}$, D. WATSON $^{4}$, \\ A. J. Castro-Tirado ${ }^{12}$, R. A. M. J. Wijers ${ }^{13}$, R. L. C. Starling ${ }^{1}$, K. Misra ${ }^{3}$, J. F. Graham ${ }^{3}$, And C. Kouveliotou ${ }^{14}$ \\ ${ }^{1}$ Department of Physics and Astronomy, University of Leicester, University Road, Leicester LE1 7RH, UK; nrt3@ star.le.ac.uk \\ ${ }^{2}$ Department of Physics, University of Warwick, Coventry CV4 7AL, UK \\ ${ }^{3}$ Space Telescope Science Institute, 3700 San Martin Drive, Baltimore, MD 21218, USA \\ ${ }^{4}$ Dark Cosmology Centre, Niels Bohr Institute, University of Copenhagen, Juliane Maries Vej 30, DK-2100 Copenhagen Ø, Denmark \\ ${ }^{5}$ HH Wills Physics Laboratory, University of Bristol, Tyndall Avenue, Bristol BS8 1TL, UK \\ ${ }^{6}$ School of Earth and Space Exploration, Arizona State University, Tempe, AZ 85287, USA \\ ${ }^{7}$ Centre for Astrophysics and Cosmology, Science Institute, University of Iceland, Dunhagi 5, 107 Reykjavík, Iceland \\ ${ }^{8}$ Astrophysics Research Institute, Liverpool John Moores University, Liverpool, UK \\ ${ }^{9}$ Department of Astronomy, Yale University, New Haven, CT 06511-208101, USA \\ ${ }^{10}$ Institute for Theory and Computation, Harvard-Smithsonian Center for Astrophysics, 60 Garden Street, Cambridge, MA, 02138, USA \\ ${ }^{11}$ Max-Planck-Institut für extraterrestrische Physik, Garching bei München, Germany \\ 12 Instituto de Astrofsica de Andalucía (IAA-CSIC), Glorieta de la Astronomía s/n, 18.008 Granada, Spain \\ ${ }^{13}$ Astronomical Institute "Anton Pannekoek," P.O. Box 94248, 1090 SJ Amsterdam, The Netherlands \\ ${ }^{14}$ Space Science Office, VP62, NASA/Marshall Space Flight Center, Huntsville, AL 35812, USA \\ Received 2012 January 26; accepted 2012 April 14; published 2012 July 5
}

\begin{abstract}
We present late-time Hubble Space Telescope (HST) imaging of the fields of six Swift gamma-ray bursts (GRBs) lying at $5.0 \lesssim z \lesssim 9.5$. Our data include very deep observations of the field of the most distant spectroscopically confirmed burst, GRB 090423, at $z=8$.2. Using the precise positions afforded by their afterglows, we can place stringent limits on the luminosities of their host galaxies. In one case, that of GRB 060522 at $z=5.11$, there is a marginal excess of flux close to the GRB position which may be a detection of a host at a magnitude $J_{\mathrm{AB}} \approx 28.5$. None of the others are significantly detected, meaning that all the hosts lie below $L^{*}$ at their respective redshifts, with star formation rates (SFRs) $\lesssim 4 M_{\odot} \mathrm{yr}^{-1}$ in all cases. Indeed, stacking the five fields with WFC3-IR data, we conclude a mean SFR $<0.17 M_{\odot} \mathrm{yr}^{-1}$ per galaxy. These results support the proposition that the bulk of star formation, and hence integrated UV luminosity, at high redshifts arises in galaxies below the detection limits of deep-field observations. Making the reasonable assumption that GRB rate is proportional to UV luminosity at early times allows us to compare our limits with expectations based on galaxy luminosity functions (LFs) derived from the Hubble Ultra-Deep Field and other deep fields. We infer that an LF, which is evolving rapidly toward steeper faint-end slope $(\alpha)$ and decreasing characteristic luminosity $\left(L^{*}\right)$, as suggested by some other studies, is consistent with our observations, whereas a non-evolving LF shape is ruled out at $\gtrsim 90 \%$ confidence. Although it is not yet possible to make stronger statements, in the future, with larger samples and a fuller understanding of the conditions required for GRB production, studies like this hold great potential for probing the nature of star formation, the shape of the galaxy LF, and the supply of ionizing photons in the early universe.
\end{abstract}

Key words: galaxies: evolution - galaxies: luminosity function, mass function

Online-only material: color figures

\section{INTRODUCTION}

The drive to locate and characterize the earliest gravitationally bound objects in the universe - the first protogalaxies, Population III and II stars and the black holes they produced-is a central theme of contemporary cosmology. It is intimately tied to the quest to understand how the universe at large was reionized, quite possibly by the ultraviolet (UV) flux produced by these first, and still enigmatic objects. Major investments of time on large facilities continue to be spent on these ambitious goals, but progress has been hard-won. Historically, quasars were the key high-redshift beacons, thanks to their great luminosities, but the space density of bright quasars drops above $z \sim 4$ and to date the most distant quasar is located at $z=7.1$ (Mortlock et al. 2011). Galaxies are, of course, much more common than quasars, and high- $z$ galaxies are likely to be present in moderate numbers in very deep near-infrared (NIR) observations. Indeed, recent NIR observations in the Hubble Ultra-Deep Field (HUDF) have unveiled $>100$ candidate $z>7$ galaxies (e.g., Bouwens et al. 2011b; Bunker et al. 2010; McLure et al. 2010) discovered via the Lyman-dropout technique. The challenge of studying these sources lies in their extreme faintness (often $>28$ th magnitude), which generally means only photometrically derived redshift estimates are possible. Furthermore, distinguishing young, high- $z$ galaxies from old or dusty populations at moderate redshifts, or even Galactic brown dwarfs, becomes increasingly difficult when approaching the sensitivity limit of the data. Although spectroscopic redshifts have been determined for a small number of $z>7$ galaxies (Vanzella et al. 2011; Ono et al. 2012), this is only possible if the galaxy is a strong Ly $\alpha$ emitter (and the emission is not completely absorbed by a partially neutral intergalactic medium (IGM)), and requires a major investment of time for each target. Even in the era of extremely large telescopes (ELTs), many such sources may be too faint for direct spectroscopic redshift confirmation.

The extreme luminosity of gamma-ray bursts (GRBs) makes them potentially powerful probes of the early universe, a utility which has been widely touted since the discovery of the first afterglows (e.g., Wijers et al. 1998; Lamb \& Reichart 2000; Tanvir \& Jakobsson 2007). Current technology has the capability to detect the prompt, and afterglow emission for bright GRBs out to $z \sim 20$, should GRBs exist at this epoch (Gou et al. 2004; 
Racusin et al. 2008; Bloom et al. 2009), and they have several advantages over alternative methods for the detection and study of high- $z$ objects. In the first instance, the intrinsically smooth power-law spectra of the afterglows makes them ideal backlights for absorption diagnostics: not only providing redshifts, but in principle chemical enrichment, hydrogen column densities (e.g., Vreeswijk et al. 2004; Prochaska et al. 2007; Fynbo et al. 2009), extinction and dust laws in the hosts (e.g., Zafar et al. 2011a; Schady et al. 2012), and even probing the state of the IGM (e.g., Miralda-Escude 1998; McQuinn et al. 2008). Second, they pinpoint the positions of their hosts, and once the afterglow has faded we can search for the host galaxy with a redshift in hand from spectroscopic (or photometric) observations of the afterglow. Finally, long-duration GRBs are produced during core-collapse events (e.g., Hjorth et al. 2003b; Stanek et al. 2003; Pian et al. 2006), and thus trace the locations of at least some component of massive star formation. Importantly, GRBs allow absorption redshift measurements for galaxies at times too faint to be seen in deep imaging even with the Hubble Space Telescope (HST; e.g., Berger et al. 2002; Hjorth et al. 2003a; Thöne et al. 2010).

The early populations of massive stars and the protogalaxies in which they reside are thought to be major producers of ionizing photons, and also give rise to GRBs. Hence, mapping GRB space density as a function of cosmic time should trace this early star formation and provide a key to assessing its contribution to the reionization of the IGM at $z>6$. Since GRB progenitors are individual stellar systems, their hosts should sample the whole (star-forming) galaxy luminosity function (LF), rather than just the bright end, avoiding the limitation inherent in flux limited samples (e.g., Jakobsson et al. 2005). This is particularly important at very high redshift, when galaxies were small and faint, and only the tip of the LF can be probed directly.

However, GRB-based studies are subject to their own difficulties. As with any sources beyond $z \sim 6$, in addition to the increasing luminosity distance, we have to contend with the difficulties of working in the NIR. In addition, because GRB afterglows fade, there is only a narrow window of opportunity in which observations can be pursued, so signal to noise cannot be built up over many nights of observation. Hence, a major constraint in their detection and study at high- $z$ is the availability of large aperture telescopes which can respond rapidly with appropriate NIR instrumentation. It is also clear that high-redshift GRBs detectable to the limits of the Swift satellite are rather rare, and therefore many bursts have to be observed to find the few at $z>5$ (e.g., Jakobsson et al. 2012). Finally, studies of GRBs at lower redshift have indicated that they are generally found in small-to-moderate size, low-to-moderate metallicity galaxies with high specific star formation rates (= SFR $/ M_{\mathrm{gal}}$; e.g., Christensen et al. 2004; Castro Cerón et al. 2006; Modjaz et al. 2008; Chen et al. 2009; Savaglio et al. 2009; Svensson et al. 2010), and rarely in intensively star-forming far-IR-bright galaxies (Tanvir et al. 2004; Le Floc'h et al. 2006; Michałowski et al. 2008). Coupled with theoretical arguments (e.g., Yoon \& Langer 2005; Woosley \& Heger 2006) and spectroscopic studies of their environments (e.g., Fynbo et al. 2009; Levesque et al. 2010a), this has led to the suggestion that GRBs may be more common and/or brighter at relatively low metallicities (e.g., Wolf \& Podsiadlowski 2007). Such a dependence could potentially help explain the apparent increase in GRB-rate to SFR ratio between $z \sim 0$ and $z \sim 5$ seen in various studies (e.g., Natarajan et al. 2005; Yüksel et al. 2008; Robertson \&
Ellis 2012). However, the picture is not a simple one: there is a selection bias against finding heavily extinguished afterglows (e.g., Perley et al. 2009; Krühler et al. 2011), and at least some GRBs appear to be produced in very dusty, massive starbursting galaxies (e.g., Chen et al. 2010; Küpcü Yoldaş et al. 2010; Hashimoto et al. 2010; Svensson et al. 2012), whilst others have been found in solar or even supersolar metallicity environments (Levesque et al. 2010b; Savaglio et al. 2011). Furthermore, comparison of GRB luminosity and host metallicity at $z<1$ reveals no obvious correlation (Levesque et al. 2010c). In any event, it is likely that galaxies with properties similar to typical GRB hosts predominate at early times, and indeed the small number of higher-redshift afterglows for which accurate metallicities have been derived show a range that matches well predictions from galaxy evolution simulations that include no bias in GRB production (Pontzen et al. 2010). Thus, we expect GRBs to be a good tracer of the bulk of high-redshift star formation (Fynbo et al. 2006, 2008; Kocevski et al. 2009), and we proceed under that assumption in this paper.

Several very high $z$ bursts have been identified by Swift to date, and have begun to realize their potential as probes of the early universe. The afterglow of GRB 050904 at $z=6.3$ was brighter than magnitude $J=17.5$ even a few hours after the burst (Haislip et al. 2006), and spectroscopy would have been routine with a 4-8 m class telescope. Indeed, ultimately the redshift measurement came from a Subaru spectrum taken some three days after the event (Kawai et al. 2006). Even the intrinsically rather faint afterglows of GRB 080913 ( $i=20$; Greiner et al. 2009) or GRB 090423 ( $J=21$; Tanvir et al. 2009) allowed for spectroscopic redshifts with $8 \mathrm{~m}$ instrumentation, despite the data being acquired more than $12 \mathrm{hr}$ after the burst in the latter case.

Until now, the most distant GRB host detected (with Spitzer) was that of GRB 060510B at $z=4.94$ (Chary et al. 2007). In this paper we present deep, late-time HST observations of the fields of six of the most distant GRBs, all at $z>5$. Only one host galaxy, that of GRB 060522, could be marginally detected, and the deep limits for the others imply that all lie close to or below the characteristic luminosity, $L^{*}$ at their respective redshifts. As we show in this paper, the non-detections of the hosts place important constraints on their total luminosities and SFRs. Since long-duration GRBs trace (at least) some component of star formation, a survey of even a small number of GRBs at high redshifts provides a census of the locations and galactic environments of star formation at early times. This means that GRBs have the potential ultimately to constrain the faint end of the galaxy LF at $z>7$, which is crucial for understanding the reionization of the universe, thought to occur predominantly at $8<z<12$ (Komatsu et al. 2011). UV photons produced by massive stars are widely considered the most likely driving force for reionization (Loeb 2009; Faucher-Giguère et al. 2009), but even ultra-deep surveys cannot currently quantify the (likely dominant) contribution of intrinsically faint galaxies.

Throughout this paper, we use the AB-magnitude system, and adopt a $\Lambda$-CDM cosmology with $H_{0}=72 \mathrm{~km} \mathrm{~s}^{-1} \mathrm{Mpc}^{-1}$, $\Omega_{M}=0.27$, and $\Omega_{\Lambda}=0.73$.

\section{GRBs IN THE SAMPLE}

Our sample comprises six of the most distant bursts detected by Swift, including all five bursts detected to date that have firm spectroscopic redshifts above $z=5$. They are GRBs 050904 $(z=6.29$; Kawai et al. 2006), $060522(z=5.11$; Cenko et al. 2006), 060927 ( $z=5.47$; Ruiz-Velasco et al. 2007), 080913 


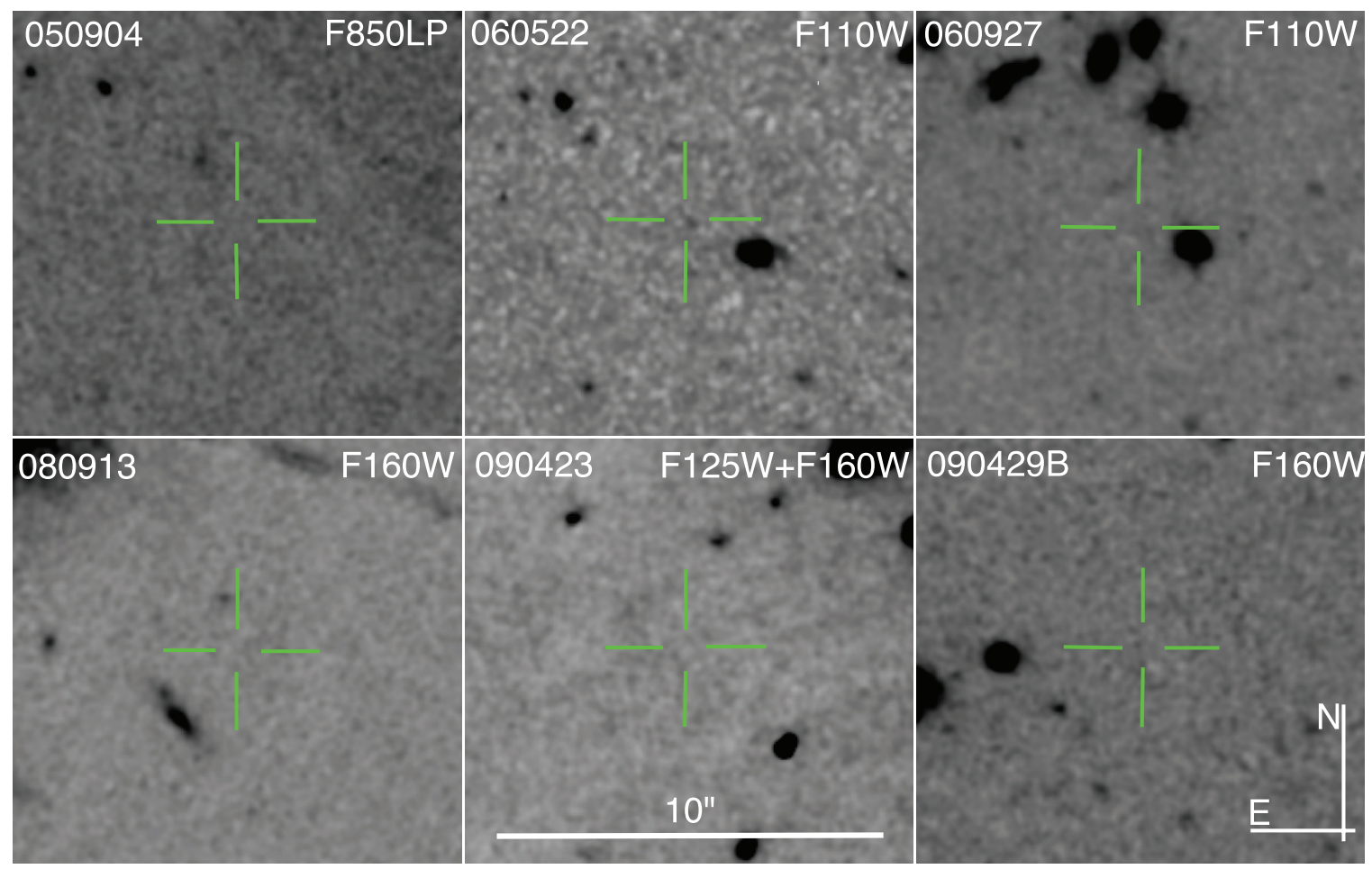

Figure 1. Mosaic of the host galaxy fields of the $z>5$ GRBs in our study. The filters of the observations are indicated in each panel, while the cross-hairs represent the location of each burst as determined from astrometry with ground-based images containing the afterglow (see Table 1 for more details). The images have been lightly smoothed to allow the eye to see fainter features.

(A color version of this figure is available in the online journal.)

Table 1

Summary of Burst Sample and Astrometric Ties

\begin{tabular}{lccllc}
\hline \hline GRB & $z$ & $z$-ref & \multicolumn{1}{c}{ Obs. Summary } & Astrometry & $\begin{array}{c}\text { Accuracy } \\
(")\end{array}$ \\
\hline 060522 & 5.11 & 1 & WFC3:F110W & TNG & 0.06 \\
060927 & 5.47 & 2 & NIC3:F160W, WFC3:F110W & VLT/FORS2 & 0.07 \\
050904 & 6.29 & 3 & ACS:F850LP & Gemini-S/GMOS & 0.06 \\
080913 & 6.73 & 4 & WFC3:F160W & VLT/FORS2 & 0.08 \\
090423 & 8.23 & 5 & WFC3:F125W, F160W & VLT/HAWK-I & 0.03 \\
$090429 \mathrm{~B}$ & $9.4^{\mathrm{a}}$ & 6 & WFC3:F105W, F160W & Gemini/NIRI & 0.06 \\
\hline
\end{tabular}

Notes.

${ }^{\text {a }}$ Photometric redshift estimate.

References. (1) Cenko et al. 2006; (2) Ruiz-Velasco et al. 2007; (3) Kawai et al. 2006; (4) Patel et al. 2010; (5) Tanvir et al. 2009; (6) Cucchiara et al. 2011.

$(z=6.73$; Greiner et al. 2009; Patel et al. 2010), 090423 $(z=8.23$; Tanvir et al. 2009; Salvaterra et al. 2009), and GRB 090429B ( $z_{\text {phot }}=9.4$; Cucchiara et al. 2011). Here, we briefly summarize the details of each GRB, and the available HST observations of each field, which were mostly obtained after the afterglow and should have faded beyond detectability (the possible exception is GRB 050904 as discussed below). The majority of our data comes from the new Wide Field Camera 3 Infrared channel (WFC3 IR). In all of these cases, we used the standard flat-fielded observations from the HST archive, ${ }^{15}$ and corrected these for geometric distortion, creating a stack of individual images using multidrizzle. The final pixel scale for these well-dithered observations is set as $0{ }^{\prime \prime} 05$ per pixel, with the pixfrac set to 0.7. For observations with Advanced Camera for Surveys (ACS), we drizzled to the same final pixel scale,

\footnotetext{
15 http://archive.stsci.edu/
}

which broadly retains the native pixel scale of the instrument. For GRB 060927, we also present NICMOS observations of the host, as detailed below. Cutouts of the images around the locations of the GRB positions are shown in Figure 1.

The exact locations of each burst on the HST images were found relative to field sources using ground-based images of their afterglows. Details of the data obtained for each burst and the rms accuracy of the astrometric calibration are summarized in Table 1.

For photometry, following Bouwens et al. (2010a), we employed 0.'4 diameter apertures centered at the locations of the afterglows (for reference, 0 .' 4 corresponds to a physical scale of $\approx 2.1 \mathrm{kpc}$ at $z=7$ ). We note this is appropriate since GRBs are generally found to be close to the UV-brightest regions of their hosts (Fruchter et al. 2006), and the sizes of high- $z$ galaxies are found to be intrinsically small (e.g., $0.7 \pm 0.3 \mathrm{kpc}$ was found for $z \approx 7-8$ galaxies in the HUDF by Oesch et al. 2010). The prior 
Table 2

Log of HST Observations of the Host Galaxies of GRBs at $z>5$

\begin{tabular}{|c|c|c|c|c|c|c|c|}
\hline Date & UT Time & Filter & $\begin{array}{l}\lambda_{\text {rest }} \\
(\AA)\end{array}$ & $\begin{array}{l}\text { Exp } \\
(s)\end{array}$ & $\begin{array}{l}F_{\mathrm{obs}} \\
(\mathrm{nJy})\end{array}$ & AB mag Limit & $M_{\lambda /(1+z)}$ \\
\hline \multicolumn{8}{|l|}{060522} \\
\hline 2010 Oct 17 & $10: 30$ & F110W & 1888 & 8395 & $7 \pm 4$ & $>28.13$ & $>-18.35$ \\
\hline \multicolumn{8}{|l|}{060927} \\
\hline 2007 Jun 29 & $11: 30$ & F160W & 2376 & 10240 & $7 \pm 5$ & $>27.75$ & $>-18.84$ \\
\hline 2010 Sep 25 & $14: 30$ & F110W & 1782 & 13992 & $4 \pm 3$ & $>28.57$ & $>-18.02$ \\
\hline \multicolumn{8}{|l|}{050904} \\
\hline 2005 Sep 26 & $21: 03$ & F850LP & $1279^{b}$ & 4216 & $-9 \pm 30^{\mathrm{b}}$ & $>26.86$ & $>-19.95$ \\
\hline \multicolumn{8}{|l|}{080913} \\
\hline 2009 Nov 30 & $16: 10$ & F160W & 1988 & 7818 & $3 \pm 6$ & $>27.92$ & $>-19.00$ \\
\hline \multicolumn{8}{|l|}{090423} \\
\hline 2010 Jan 24 & $11: 34$ & F160W & 1665 & 13029 & & & \\
\hline $2010 \operatorname{Jan} 25^{\mathrm{a}}$ & $14: 44$ & F160W & 1665 & 13029 & $4 \pm 3$ & $>28.36$ & $>-18.88$ \\
\hline $2010 \operatorname{Jan} 26^{\mathrm{a}}$ & $13: 06$ & F125W & 1353 & 13029 & & & \\
\hline $2010 \operatorname{Jan} 27^{\mathrm{a}}$ & 13:04 & F125W & 1353 & 13029 & & & \\
\hline 2010 Oct 22 & $18: 23$ & F125W & 1353 & 13029 & & & \\
\hline 2010 Oct 27 & $16: 36$ & F125W & 1353 & 13029 & $-2 \pm 2$ & $>30.29$ & $>-16.95$ \\
\hline \multicolumn{8}{|l|}{ 090429B } \\
\hline 2010 Jan 10 & $21: 54$ & F160W & 1478 & 2412 & & & \\
\hline 2010 Feb 22 & $19: 22$ & F160W & $\begin{array}{r}1478 \\
(2049)\end{array}$ & 2412 & $7 \pm 5$ & $>27.78$ & $\begin{array}{c}>-19.65 \\
(>-19.09)^{\mathrm{c}}\end{array}$ \\
\hline 2010 Feb 24 & 03:19 & F105W & 1014 & 2412 & & & \\
\hline 2010 Feb 28 & $13: 56$ & F105W & $\begin{array}{r}1014 \\
(1407)\end{array}$ & 2412 & $-1 \pm 5$ & $>28.49$ & $\begin{array}{c}>-18.73 \\
(>-18.17)^{\mathrm{c}}\end{array}$ \\
\hline
\end{tabular}

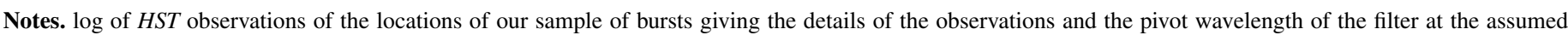

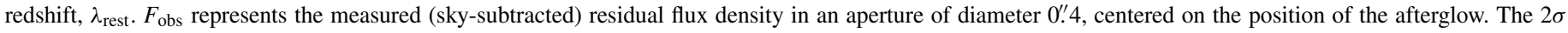
point-source limits on apparent magnitude (after aperture correction) and corresponding absolute magnitude for each filter are also given.

a Images affected by persistence.

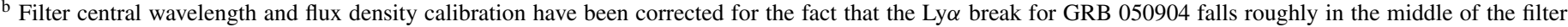

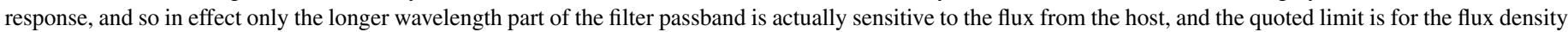
redward of Ly $\alpha$.

${ }^{\mathrm{c}}$ Result if GRB 090429B was actually at the lower limit for its photometric redshift of $z \approx 6.5$.

knowledge of the exact locations of the GRBs allows us to adopt a $2 \sigma$ excess flux as a reasonable detection threshold, which is unlike blind searches where a much higher level of significance is required to ensure confidence that the source is real and also to reliably constrain the photometric redshift. For comparison with galaxy samples, and GRB hosts at other redshifts, it is relevant to consider the appropriate aperture corrections, ${ }^{16}$ and these are included in the limits shown in Table 2. Our errors are determined in each case from the variance within a set of apertures of equal size to our source aperture, placed at random blank sky locations in the field surrounding the source.

The results of the photometric analysis, and inferred limits on host properties, are given in Table 2. Note that we report the measured sky-subtracted flux densities even when they are not significant detections, or indeed are formally negative. This is to allow comparison with models, as is done in Section 3.

\subsection{GRB 060522}

Spectroscopy of GRB 060522 was performed by Keck roughly $12.5 \mathrm{hr}$ after the burst, and revealed a strong break

\footnotetext{
16 That is, the limits in this table are given by $M_{\lim }=23.9-2.5 \mathrm{log}$ (flux $+2 \sigma)+a p_{\text {corr }}$. The aperture corrections are calculated from the encircled energy (e.g., http://www.stsci.edu/hst/wfc3/documents/handbooks/currentIHB/ c07_ir07.html) and have values for WFC3/IR of ap $105=0.31$, ap $_{110}=$ $0.33, \mathrm{ap}_{125}=0.39, \mathrm{ap}_{160}=0.52$, for ACS $/ \mathrm{WFC} \mathrm{ap}_{850}=0.27$ (Sirianni et al. 2005), and for NICMOS/NIC3 ap $160=0.60$. http://www.stsci.edu/hst/nicmos/ documents/handbooks/current_NEW/c04_imaging.6.5.html
}

$\sim 7425 \AA$ in the spectrum, interpreted as $\operatorname{Ly} \alpha$ at $z=5.11 \pm 0.01$ (Cenko et al. 2006). A search for the host galaxy with the Spitzer Space Telescope found no detection to flux densities of $0.2 \mu \mathrm{Jy}$ at $3.6 \mu \mathrm{m}$ and $2.4 \mu \mathrm{Jy}$ at $5.8 \mu \mathrm{m}$, implying that its rest-frame optical luminosity lies substantially below $L^{*}$ (Chary et al. 2007).

We obtained observations of GRB 060522 with WFC3/IR using the F110W filter. To ascertain the precise location of the burst on our HST image, we performed relative astrometry between our WFC3 observations and an image obtained at the Telescopio Nazionale Galileo (TNG) on 2006 May 22 (D'Avanzo et al. 2006). At the location of the afterglow, we measure a flux density of $7 \pm 4 \mathrm{nJy}$, corresponding to a $2 \sigma$ (aperture corrected) magnitude limit of $\mathrm{F} 110 \mathrm{~W}(\mathrm{AB})>28.13$. Although formally this is a non-detection at $2 \sigma$ it is possible that some host flux is contributing within the aperture. Indeed visually it appears there is a somewhat more significant excess of flux which is offset slightly southwest from the GRB location (by about 0.3 , or $\sim 2 \mathrm{kpc}$ at $z=5.11$ ), and if we increase the aperture size to 0'. 6 diameter then the flux density becomes $12 \pm 5 \mathrm{nJy}$. This would represent a marginal detection, and corresponds to an apparent magnitude of $\mathrm{F} 110 \mathrm{~W}(\mathrm{AB}) \approx 28.5$. While this is a plausible magnitude for a host (corresponding to $M_{1800}(\mathrm{AB}) \approx-18$ ), the probability of a chance alignment with an unrelated object at such faint magnitude levels is also non-negligible. Specifically, considering the F110W number 
galaxy number counts from Thompson et al. (1999), we estimate $\approx 5 \%$ of random locations will have a galaxy of magnitude $\mathrm{F} 110 \mathrm{~W}(\mathrm{AB}) \lesssim 28.5$ within 0 .'5. Thus, in a sample of six, as we have, there is a $\sim 25 \%$ chance of an unrelated galaxy being close enough to be possibly mistaken for a host in at least one case. For the sake of consistency with the other bursts, we will continue to use the $00^{\prime \prime} 4$ aperture limit in the remainder of the paper.

\subsection{GRB 060927}

GRB 060927 was detected by Swift, and its optical afterglow was initially found in rapid, but unfiltered, Rapid Optical Transient Source Experiment (ROTSE) observations. An extensive follow-up campaign (reported in Ruiz-Velasco et al. 2007) revealed the source to be an $R$-band dropout, well detected in the $i$ band. A subsequent spectrum obtained at the Very Large Telescope (VLT) showed a faint continuum redward of $\sim 8000 \AA$, as well as a weak $\mathrm{Si}$ iI absorption feature, consistent with a redshift of $z=5.47$.

Our HST/NICMOS observations of the field of GRB 060927 were taken using the F160W filter, and were reduced as described in Fynbo et al. (2005). GRB 060927 was additionally re-observed with WFC3/IR using the F110W filter. Astrometry was performed via observations made with the VLT on 2006 September 30. To establish the position on the NICMOS images (which have a narrower field of view than WFC3), we opted to perform relative astrometry directly to the WFC3 images. The resulting rms astrometric accuracy is $<0^{\prime \prime} .01$, and so it does not impact the overall error in the astrometric solution described above.

At the location of the afterglow, the photometry in each of the NICMOS and WFC3/IR observations yields limiting magnitudes of $\mathrm{F} 160 \mathrm{~W}(\mathrm{AB})>27.75$ and $\mathrm{F} 110 \mathrm{~W}(\mathrm{AB})>28.57$, respectively.

\subsection{GRB 050904}

GRB 050904 was the first GRB at $z>6$ to be located. Its optical/IR afterglow was initially found by Haislip et al. (2006), who derived a photometric redshift of $z=6.39$ based on the strong spectral break between $i$ and $z$ bands, coupled with NIR observations showing a blue spectral slope redward of the break (see also Tagliaferri et al. 2005). The afterglow was intrinsically extremely bright, among the brightest observed for any burst, making late-time spectroscopy feasible with Subaru. This provided a measurement of the absorption redshift, hydrogen column density, and metallicity of the host (Kawai et al. 2006; Totani et al. 2006). It remains the most distant burst for which all of these diagnostics are available.

A search for the host galaxy of GRB 050904 was conducted by Berger et al. (2007) using both HST and Spitzer, which placed deep limits on any host emission. The first HST epoch, at which time both ACS/F850LP and NICMOS/F160W images were obtained, was carried out only about three weeks postburst. No significant flux was detected in F850LP, and the faint detection in the F160W image was shown to be due to residual afterglow contamination since it was absent in a later F160W epoch (Berger et al. 2007).

For our analysis, we re-reduced the ACS data for GRB 050904 (since we are primarily interested in the rest-frame UV luminosity close to $\operatorname{Ly} \alpha$, this filter is the more relevant). Astrometric tying of the afterglow to field sources was done utilizing a $z$-band image obtained from Gemini-South on 2005 September 7 . At the location of the afterglow in the F850LP frame, we measure a flux density of $-5 \pm 17 \mathrm{nJy}$, corresponding to a $2 \sigma$ limit of $\mathrm{F} 850 \mathrm{LP}(\mathrm{AB})>27.50$ (or 27.06 at $3 \sigma$ ). This is in good agreement with the limits reported by Berger et al. (2007). However, since the Ly $\alpha$ break lies within the filter bandpass at this redshift, we must account for flux lost due to IGM absorption (i.e., the effective filter width is narrower for this host), and so we conclude a corrected magnitude limit of F850LP(AB) > 26.86 .

\subsection{GRB 080913}

GRB 080913 was identified as a high-redshift candidate based on photometric observations with GROND showing the burst to be an $i$-band dropout (Rossi et al. 2008). Deep, red spectroscopy from the VLT showed a strong spectral break, interpreted as Ly $\alpha$ at $z \sim 6.7$ (Greiner et al. 2009). A further detailed analysis of the spectrum by Patel et al. (2010) revealed a single absorption line of Si II at $z=6.733$, and we adopt this as the redshift of GRB 080913.

We obtained HST observations with WFC3/IR in the F160W filter. To tie the astrometry of our HST observations, we utilized images obtained from FORS2 at the VLT on 2008 September 13 in the $z$ band, and images taken with NIRI on Gemini-North on 2008 September 14. We used two images independently to confirm the precise location of GRB 080913 on our HST images. For the FORS2 images, we identified nine compact sources in common, while only six sources were usable from the Gemini observations. Although the afterglow detections are of low signal-to-noise ratio $(\mathrm{S} / \mathrm{N})$, the errors on their centroids are small in comparison to the errors derived from the fit and hence we are able place the afterglow to an rms accuracy of 0 '.08. Again, no host galaxy is visible in our observations to a limit of $\mathrm{F} 160 \mathrm{~W}(\mathrm{AB})>27.92$.

\subsection{GRB 090423}

GRB 090423 was first identified as a candidate high-redshift object based on its afterglow being a $Y$-band dropout, which implies $z>7.5$ (Cucchiara et al. 2009). VLT spectroscopy with both ISAAC and SINFONI allowed the identification of the Lyman break, despite low $\mathrm{S} / \mathrm{N}$, establishing the redshift $z=8.23 \pm 0.07$ (Tanvir et al. 2009). This value is in excellent agreement with the $z=8.1_{-0.3}^{+0.1}$ determined from a spectrum obtained at the TNG (Salvaterra et al. 2009).

We obtained our first HST observations of GRB 090423 on 2010 January 24. At this stage, we acquired 20 orbits in each of the F125W and F160W filters on WFC3. Unfortunately, 15 of these 20 orbits were substantially impacted by persistence from earlier observations of bright field sources. This affected the sensitivity over wide regions of the detector, although it does allow us to measure the flux at the location of the GRB host (which was only mildly affected by persistence). Additional observations were obtained in 2010 October. All of the available observations in each filter were co-aligned and stacked via multidrizzle. These observations are extremely deep, with limiting magnitudes a factor $1.5 \times$ deeper than the WFC3 Early Release Observations of the Chandra Deep Field South/Great Observatories Origins Deep Survey (CDF-S/GOODS) fields, and only another factor of $1.5 \times$ shallower than the WFC3 observations of the HUDF (Bouwens et al. 2011b).

The location of the afterglow on our HST images was achieved by tying the astrometry to VLT/HAWK-I observations that were obtained approximately $17 \mathrm{hr}$ post-burst. While the afterglow had faded since its first discovery from Hawaii, these deep 
Table 3

Assumed Luminosity Function Parameters at Each Redshift, and Derived Host Properties and Probabilities

\begin{tabular}{|c|c|c|c|c|c|c|c|c|c|}
\hline Burst & $z$ & $M^{*} \mathrm{UV}, \mathrm{AB}$ & $\alpha$ & $m^{*}$ & $\begin{array}{c}F^{*} \\
(\mathrm{nJy})\end{array}$ & $L_{\mathrm{host}} / L^{*}$ & $\begin{array}{c}\mathrm{SFR} \\
\left(M_{\odot} \mathrm{yr}^{-1}\right)\end{array}$ & $P_{2 \sigma}$ & $\begin{array}{c}Y \\
\left(F_{\text {obs }}\right) \\
\end{array}$ \\
\hline 060522 & 5.11 & -20.59 & 1.80 & 26.22 & 118 & $<0.09$ & $<0.88$ & 0.55 & 0.47 \\
\hline 060927 & 5.47 & -20.49 & 1.81 & 26.43 & 97 & $<0.08$ & $<0.65$ & 0.55 & 0.43 \\
\hline 050904 & 6.29 & -20.26 & 1.85 & 26.83 & 67 & $<0.59$ & $<4.1$ & 0.94 & 0.28 \\
\hline 080913 & 6.73 & -20.14 & 1.88 & 27.33 & 43 & $<0.21$ & $<1.3$ & 0.82 & 0.44 \\
\hline 090423 & 8.23 & -19.72 & 1.95 & 27.97 & 24 & $<0.09$ & $<0.38$ & 0.78 & 0.30 \\
\hline 090429B & $\begin{array}{c}9.4 \\
(6.5)^{\mathrm{a}}\end{array}$ & $\begin{array}{c}-19.39 \\
(-20.20)\end{array}$ & $\begin{array}{c}2.01 \\
(1.87)\end{array}$ & $\begin{array}{c}28.59 \\
(26.68)\end{array}$ & $\begin{array}{r}13 \\
(77)\end{array}$ & $\begin{array}{c}<0.78 \\
(<0.13)\end{array}$ & $\begin{array}{c}<2.4 \\
(<0.84)\end{array}$ & $\begin{array}{c}0.96 \\
(0.71)\end{array}$ & $\begin{array}{c}0.82 \\
(0.46)\end{array}$ \\
\hline
\end{tabular}

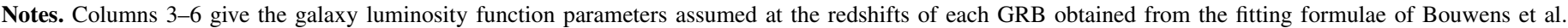

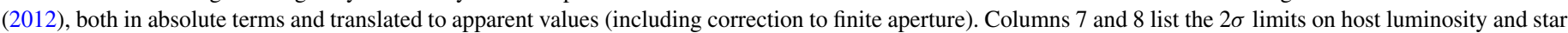

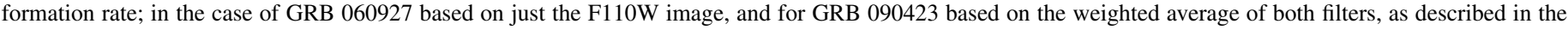

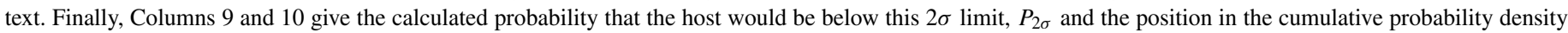

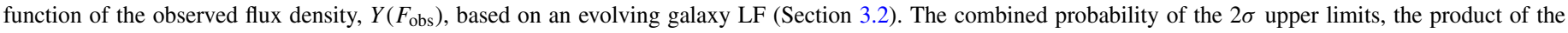
figures in Column 9, is $\prod P_{2 \sigma}=0.17$.

${ }^{\text {a }}$ Result if GRB 090429B was actually at the lower limit for its photometric redshift of $z \approx 6.5$.

observations yielded similar signal to noise, with the added advantages of a larger field of view, and a greater number of faint sources for comparison.

Within the HST image there is no obvious source at the afterglow location, and the measured $2 \sigma$ limiting magnitudes are $\mathrm{F} 125 \mathrm{~W}(\mathrm{AB})>30.29$ and $\mathrm{F} 160 \mathrm{~W}(\mathrm{AB})>28.36$. Since both filters sample the rest-frame far-UV, we form a weighted average of the two results to provide a combined flux density measure of $-0.15 \pm 1.7 \mathrm{nJy}$, which is used to derive the limit on the SFR reported in Table 3. Such a small host is consistent with the non-detection of molecular gas from the GRB location (Stanway et al. 2011).

\subsection{GRB 090429B}

GRB 090429B is the only host within our sample that does not have a spectroscopically measured redshift. However, the photometric break between the $J$ and $H$ bands, coupled with the blue spectral slope between $H$ and $K$ provides a best-fit photometric redshift of $z=9.4$ and a robust lower limit to the redshift of $z \gtrsim 6.5$ (Cucchiara et al. 2011). As we commented above, the smooth power-law spectra of GRB afterglows make photometric redshift estimates generally more reliable than they are for galaxies, since the range of intrinsic spectral variation is much less (e.g., Krühler et al. 2011). We obtained observations in F606W (ACS), F105W, and F160W; the non-detection of a host galaxy in these images, including the blue filters, provides additional support for the high- $z$ origin for this burst, since the hosts of GRBs at $z<3$ have so far always been detected in HST optical imaging. Here, we consider primarily the F160W observation, since this is the only filter redward of $\operatorname{Ly} \alpha$ at the best-fit redshift $z=9.4$. However, for completeness we also report results for the $z \approx 6.5$ lower limit, which allows us to use both the F105W and F160W data.

We ascertained the location of the burst on the HST images via relative astrometry between our first epoch $K$-band observations and those obtained with $H S T$. The $2 \sigma$ limiting magnitude at this location is $\mathrm{F} 160 \mathrm{~W}(\mathrm{AB})>27.78$. This is shallower than for the majority of the bursts in our sample, since only a two-orbit exposure was obtained. However, despite this the image still probes to faint limits comparable to the likely characteristic galaxy luminosity, $L^{*}$, at $z=9.4$.

\section{ANALYSIS AND DISCUSSION}

\subsection{Limits on Host Properties}

\subsubsection{Star Formation Rates}

At the redshifts of the bursts in question, our NIR observations probe rest-frame wavelengths roughly in the range $1300-2000 \AA$ A Limits on the UV luminosity at these wavelengths provide direct constraints on the host SFRs. A potentially important consideration in the UV is the effect of extinction by dust, which could lead to a significant underestimate of the true SFR if uncorrected. However, observational constraints from GRB afterglows at high redshifts (Greiner et al. 2009; Tanvir et al. 2009; Zafar et al. 2011b) suggest that extinction corrections are likely to be small. Similarly the blue colors of the $z \sim 7$ candidate galaxies identified in the deep HST fields (e.g., Bouwens et al. 2010b; Finkelstein et al. 2011; but, see also McLure et al. 2011) also argue for little dust in most early star-forming galaxies. We therefore assume dust extinction can be neglected.

Following Madau et al. (1998), we estimate the SFRs based on the UV luminosity at $\sim 1500 \AA$ (see also the discussion in Bunker et al. 2010):

$$
\mathrm{SFR}=\frac{L_{1500, U V}}{8 \times 10^{27} \mathrm{erg} \mathrm{s}^{-1} \mathrm{~Hz}^{-1}} M_{\odot} \mathrm{yr}^{-1} .
$$

As discussed in Section 2.1, there is a marginal detection of what may be the host of GRB 060522 at $z=5.11$, slightly offset from the burst position, but otherwise none of the hosts are significantly detected. The inferred $2 \sigma$ limits on their SFRs are given in Table 3, and in Figure 2 are plotted as a cumulative histogram of upper limits, compared to the SFRs for a sample of $z \sim 7$ HUDF galaxies from Bouwens et al. (2011b). The limits span the range 0.4-4 $M_{\odot} \mathrm{yr}^{-1}$, and indicate that the total SFRs in these galaxies are modest. For comparison, the median SFR for GRB hosts at $z \lesssim 1$ are found to be around $1-2 M_{\odot} \mathrm{yr}^{-1}$ (Svensson et al. 2010).

\subsubsection{Stacked Limits on Host Galaxy Emission}

The observations of six high- $z$ fields provide the opportunity to stack the resulting data in an attempt to provide either a combined detection, or composite limit on the observed host luminosity. However, this is complicated by the use 


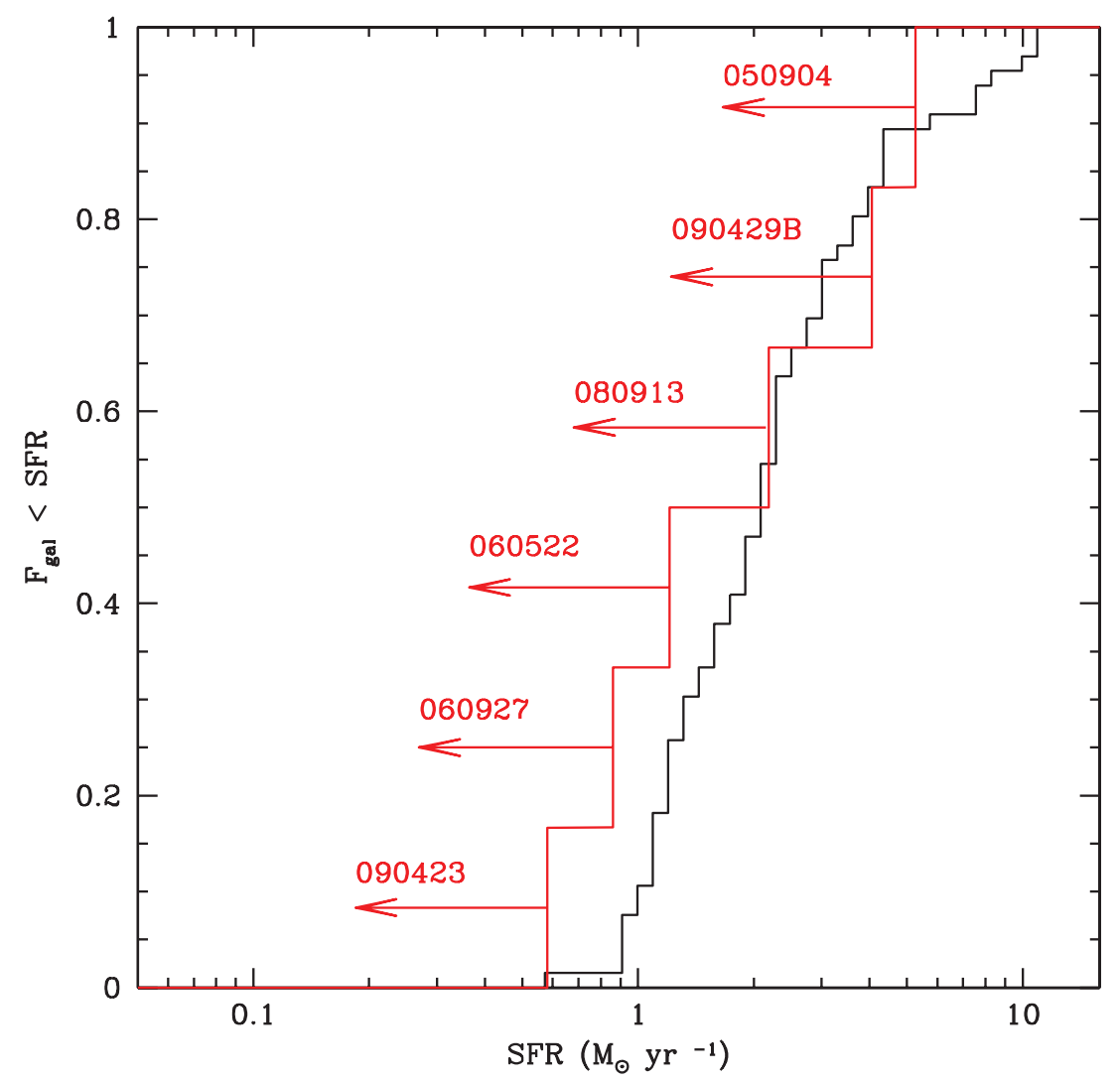

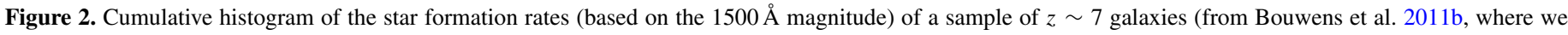

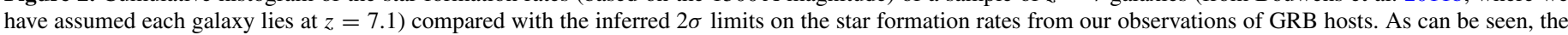

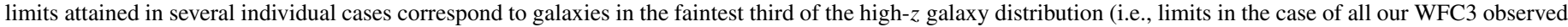
hosts). This confirms that much high- $z$ star formation is taking place in relatively faint galaxies, too faint to be found in most flux limited surveys.

(A color version of this figure is available in the online journal.)

of different instrument and filter combinations to obtain the imaging. Therefore, we do not attempt to include data taken with different instruments, and consider a stack of only the WFC3 observations. Thus, we omit the observations of GRB 050904, and the NICMOS observations of GRB 060927. To perform the stack, we first re-scaled the individual images (taken in different filters) such that the units of each image can be considered equivalent. We then computed the necessary offsets to overlay the burst positions, and re-drizzled onto a single output frame with these offsets applied. Simple aperture photometry at this location was then performed, with our errors based on the scatter in background apertures as before. The mean luminosity distance is $68,500 \mathrm{Mpc}$, corresponding to a mean redshift of $z=6.82$. There is no significant excess flux at the afterglow location, and formally the measured stacked flux density is $1 \pm 3 \mathrm{nJy}$. Hence, the mean flux density of each host galaxy is constrained to be $0.2 \pm 0.6 \mathrm{nJy}$, corresponding to a $2 \sigma$ limit of $m_{\mathrm{AB}}>30.7$, equivalent to an absolute magnitude $M_{\lambda /(1+z)}<-16.2$ and SFR $<0.17 M_{\odot} \mathrm{yr}^{-1}$, at this mean redshift.

\subsection{Constraints on High-redshift Galaxy Luminosity Functions}

Although our small sample of high- $z$ GRBs does not yet allow us to place strong constraints on the galaxy LF, we can test whether the limits on the host magnitudes are consistent with them having been drawn from the LFs suggested by other studies of galaxy populations with redshifts between 5 and 10 .
The recent re-observations of the HUDF with WFC3/IR have revealed a population of $z$-band and $Y$-band dropouts, with colors consistent with galaxies at $z>7$ and $z>8$, respectively (Bunker et al. 2010; McLure et al. 2010; Bouwens et al. 2010a). A single candidate $z \sim 10$ galaxy ( $J$-band dropout) has also been identified (Bouwens et al. 2011a). From these samples, assuming they are substantially complete and uncontaminated, it is possible to make some statements about the form and evolution of the galaxy LF from $5<z<10$. In particular, these authors fit their data in bins of redshift with the LFs described by a Schechter function (Schechter 1976):

$$
\phi(x) d x=\phi^{*} x^{-\alpha} e^{-x} d x,
$$

where $x=L / L^{*}$, with $L^{*}$ being the characteristic luminosity of the "knee" of the LF. Here, $\alpha$ is the power-law slope toward faint luminosities and $\phi^{*}$ is a normalization factor. Of course, there are no strong observational reasons to expect the LF to have this form at high- $z$, but it is supported by theoretical work (Trenti et al. 2010).

In Figure 3, we show the preferred analytical LF fits of Bouwens et al. (2011b) and the data on which they are based, for samples at $z \sim 5,7$, and 8. Their conclusion is that the characteristic knee in the LF, $L^{*}$, becomes slowly fainter with increasing redshift from 4 to 8 , and at the same time the faint-end slope becomes steeper, reaching a value of $\alpha \sim 2$ by $z=8$. This is in addition to the overall normalization, $\phi^{*}$, lowering (but see also McLure et al. 2010, who find a fading $L^{*}$ and lower $\phi^{*}$ but less evidence for a steepening 


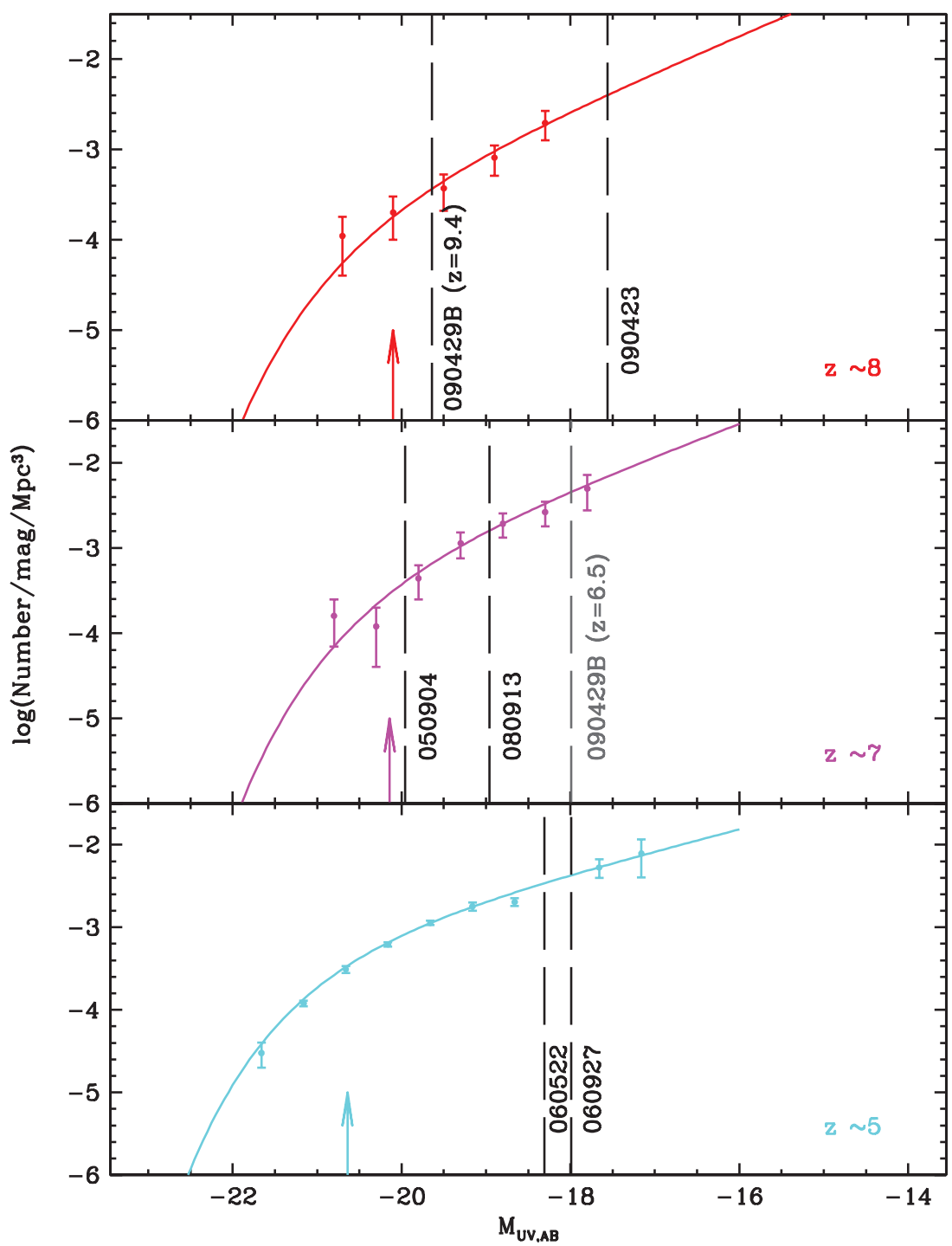

Figure 3. Our $2 \sigma$ upper limits on the magnitudes our individual host galaxies (vertical dashed lines), compared to the luminosity functions of galaxies at $z=5,7,8$ (solid curves), as determined by Bouwens et al. (2011b). In particular, for GRB 090423, the power of deep HST observations to probe sources at known locations and redshifts to well below $M^{*}$ (indicated by upward arrows) can be readily appreciated. Note that GRB 090429B is plotted both in the top, highest redshift, panel appropriate for its best-fit photometric redshift, and also (lighter shade) in the middle, intermediate redshift, panel appropriate for the lower limit of photo- $z \approx 6.5$ (see the text for more details).

(A color version of this figure is available in the online journal.)

slope). This indicates that an increasing proportion of star formation is occurring in fainter galaxies, and indeed formally the integrated luminosity represented by a Schechter function with $\alpha>2$ diverges without some lower cutoff luminosity. However, there are important caveats which pertain to these analyses: first, the LF parameters are based on entire samples, and any incompleteness or contamination (particularly difficult to rule out at the faint end) will introduce biases; second, even the HUDF is limited to finding galaxies in the top few magnitudes of the LF, so that the conclusions about total SFR (and hence the production rate of ionizing photons) are sensitive to the untested assumption that a Schechter function is the appropriate form, and to the large uncertainties on the measurement of the faint-end slope.

We note that at high redshift the UV LF has limitations as a way of representing the whole population of galaxies, since they are typically only visible when in the starbursting phase, and likely remain in this state for only a short duration based on the typical ages, and apparent availability of molecular gas for star formation within them. However, particularly for understanding the contribution of galaxies to the reionization of the universe, the measured UV luminosities, which are more representative of star formation occurring with the last $\sim 15-100 \mathrm{Myr}$, are the relevant quantities. Furthermore, since the GRB itself is a sign of ongoing massive star formation within the host, one would expect the likelihood that a GRB occurs to scale with the UV luminosity. Indeed, we would expect the region immediately underneath the burst to be extremely UV-bright, as is observed in the more local GRB population (Fruchter et al. 2006). Hence, we can meaningfully compare our limits on host emission with the observed galaxy LFs at $z \sim 7$.

The $2 \sigma$ luminosity limits for the GRB hosts are also shown graphically in relation to the high- $z$ galaxy LFs in Figure 3, while their tabulated UV absolute magnitude limits are given in Table 2. As can be seen, all the hosts are apparently below $M^{*}$ (the $\mathrm{AB}$ magnitude corresponding to $L^{*}$ ) at their respective 
redshifts, and in the case of our observations of GRB 090423, have the ability to probe to fainter limits than has so far been possible with the Ultra-Deep Field observations. This is because, despite the shallower depth of our images, we have prior knowledge that an object exists at this location, and so can accept a lower formal significance level for detection (since the chances of a random noise fluctuation are lower due to the much smaller area under consideration). Furthermore, since we do not need a blue "veto" filter we make more efficient use of the available exposure time, in contrast to some Lyman break searches which are limited by the depth of their shortwavelength (blueward of $\mathrm{Ly} \alpha$ ) imaging.

These observations of GRB host fields allow us to test the validity of the galaxy LFs that have been derived from deepfield observations. We assume that there is no dependence of GRB rate or luminosity on environmental parameters such as metallicity, which, as discussed in Section 1, is certainly plausible at high redshifts when the bulk of star-forming galaxies were generally not highly enriched. We also assume that any dust extinction is minor, as argued in Section 3.1.1. Then the probability that a galaxy produces a GRB in some unit time (the probability of more than one GRB in realistic observing times being negligible) is approximately proportional to its rest-frame UV flux, since massive stars are responsible for producing both: i.e., $P_{\mathrm{GRB}} \propto \mathrm{SFR} \propto L_{\mathrm{UV}}$. Thus, because we would expect GRBs to be drawn randomly from the total stellar UV luminosity, this means that the host galaxies should be drawn from the luminosity-weighted galaxy LF.

In practice, the best-fit values for the faint-end slope $\alpha$ and characteristic luminosity $L^{*}$ at each redshift considered were determined from the fitting formulae given by Bouwens et al. (2012), and are summarized in Table 3 . We can then calculate a luminosity-weighted LF, which (suitably normalized) we take to be equivalent to a probability density function (PDF) for the intrinsic host luminosity:

$$
y(L)=\frac{L \phi_{z}(L)}{\int_{L_{\min }}^{\infty} L^{\prime} \phi_{z}\left(L^{\prime}\right) d L^{\prime}}
$$

where $\phi_{z}(L)$ is the LF at redshift $z$. We emphasize again that, since we are going to compare with the fluxes measured in apertures at the exact GRB location, we are also assuming there is no significant offset between the GRB and its parent galaxy.

Hence, the probability of observing a GRB in a galaxy of luminosity $L<L_{\text {host }}$ can be obtained via the cumulative probability density function (CDF):

$$
Y\left(L_{\mathrm{host}}\right)=P\left(L<L_{\mathrm{host}}\right)=\frac{\int_{L_{\min }}^{L_{\mathrm{host}}} L^{\prime} \phi_{z}\left(L^{\prime}\right) d L^{\prime}}{\int_{L_{\min }}^{\infty} L^{\prime} \phi_{z}\left(L^{\prime}\right) d L^{\prime}} .
$$

Setting a lower limit, $L_{\min }$, for the integral is physically motivated since small dark halos $\left(\lesssim 10^{8} M_{\odot}\right)$ at $z \sim 10$ are expected to retain little gas and form few stars (Read et al. 2006). Given the apparent steepness of the faint-end slope of the LF at $z \sim 8$, it is also important that the value of $L_{\min }$ be sensibly chosen. It is not feasible to directly measure this lower limit at high redshift since it is well below the detection threshold for deep imaging, and will remain so even in the era of the James Webb Space Telescope (JWST) or ground-based ELTs. However, it is possible to provide estimates via simulations (Read et al. 2006), or from the star formation histories of the lowest mass galaxies in the local universe (e.g., Weisz et al. 2011). This latter approach suggests that the lowest mass galaxies attain total stellar masses of $\sim 10^{6} M_{\odot}$, over a Hubble time, implying mean SFRs of $\sim 10^{-5}-10^{-4} M_{\odot} \mathrm{yr}^{-1}$. However, star formation is likely to be episodic, and characterized by periods when the SFR is markedly higher than this average, and other times when the star formation is inactive, and the galaxy is effectively invisible in the UV. Indeed, the study of Weisz et al. (2011) suggests that the SFR of local dwarfs was somewhat higher in the early universe than their average SFR over the Hubble time. Based on this, we adopt a cutoff value of $L_{\text {min }}=4 \times 10^{23} \mathrm{erg} \mathrm{s}^{-1} \mathrm{~Hz}^{-1}$ which is equivalent to $M_{\mathrm{AB}}=-10$, and is similar to that considered by other recent studies (Bouwens et al. 2012; Kuhlen \& Faucher-Giguère 2012). This corresponds to an SFR of $\approx 5 \times 10^{-4} M_{\odot} \mathrm{yr}^{-1}$. We note that when $\alpha<2$ (i.e., for redshifts less than $z \sim 7$ ) the precise choice of $L_{\min }$ has little impact on the results.

\subsubsection{Analysis $I$}

We now consider this question: what is the probability that each host individually is fainter than the $2 \sigma$ upper bound we have inferred for its luminosity? To this end, we calculate $P_{2 \sigma}$ for each host by setting $L_{\text {host }}$ in Equation (4) equal to this upper bound.

The results are summarized in Table 3 and illustrated in Figure 4, which shows the CDFs for luminosity (expressed relative to $L^{*}$ at the redshift in question) of two bursts from our sample, together with the $2 \sigma$ detection limits for the corresponding HST frames. We see that, given the above assumptions, it is not surprising to find individual hosts to be undetected since the majority of the likelihood lies below these $2 \sigma$ bounds. However, the joint probability that none of the hosts are detected is only $P$ (none) $=\prod P_{2 \sigma}=0.17$. While not a highly significant result, this does suggest that a non-evolving LF, in which more star formation was taking place in galaxies with luminosities around $L^{*}$, would not sit comfortably with the apparent faintness of the GRB hosts.

\subsubsection{Analysis II}

A weakness of the above approach is that it does not make full use of the joint probability of the formally measured fluxes at the positions of the whole sample of GRB hosts. As an alternative, we perform the following analysis, again with the aim of testing the evolving galaxy LFs proposed by Bouwens et al. (2012).

As before, we construct a CDF for host luminosity, but now turn this into an equivalent $\mathrm{CDF}$ for observed flux density $F$ using the cosmological luminosity distance for the given redshift, having accounted for the aperture corrections. The next step is to convolve this with the observational errors appropriate for the given GRB field observation, in order to obtain a CDF for the observed host galaxy flux density:

$$
Y(F)=\frac{\int_{F_{\min }}^{F_{\mathrm{obs}}} G *\left(F^{\prime} \phi_{z}\left(F^{\prime}\right)\right) d F^{\prime}}{\int_{F_{\min }}^{\infty} G *\left(F^{\prime} \phi_{z}\left(F^{\prime}\right)\right) d F^{\prime}},
$$

where $G$ is a Gaussian with a width $\sigma$ dictated by the sky noise in each image, measured from numerous sky apertures of equal radius to the source aperture. $F_{\text {obs }}$ is the formal flux measurement at the location of the GRB, and the minimum flux density, $F_{\min }$, is appropriately scaled from the minimum luminosity, discussed above.

For illustration, the CDFs for two of our bursts are shown in Figure 5 (red curves), along with the CDFs for true host flux for comparison (blue curves). The green lines indicate the 

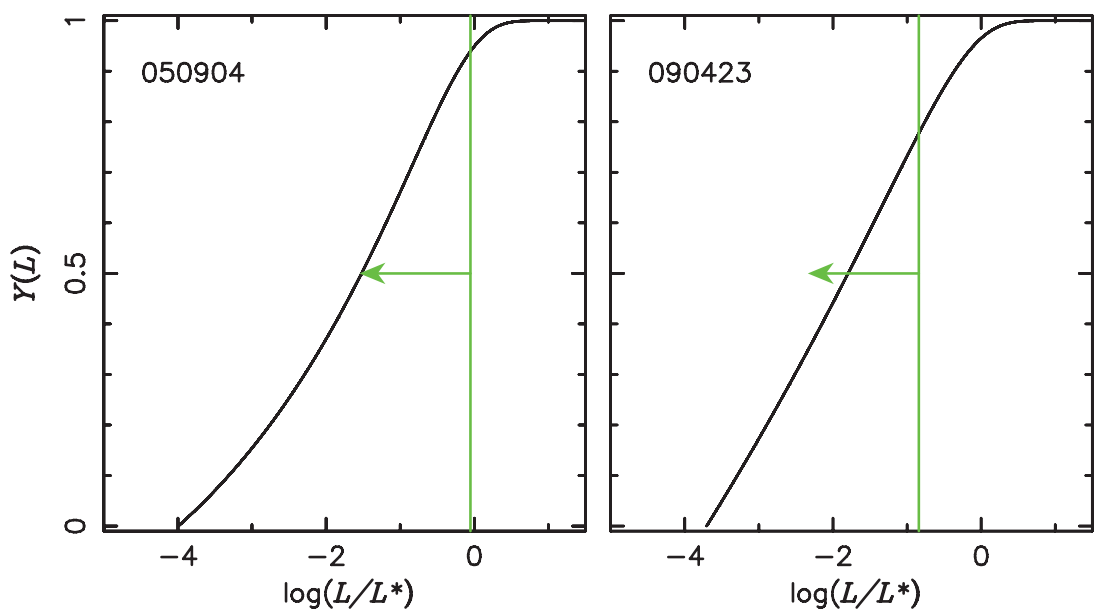

Figure 4. These panels show the cumulative probability distributions for the luminosities of two of our GRB hosts, based on assuming GRB likelihood is proportional to UV luminosity, as described in Section 3.2. Galaxy LF parameters are taken from Bouwens et al. (2012). The vertical lines correspond to the $2 \sigma$ detection limits for our HST images. As noted, in no case do we detect a host at this level of confidence.

(A color version of this figure is available in the online journal.)
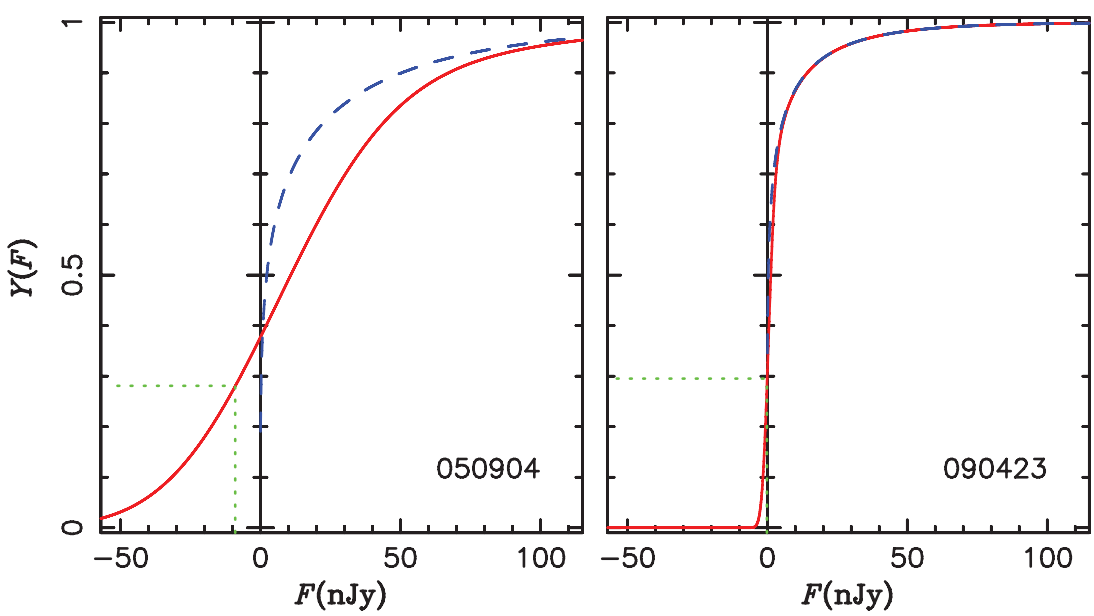

Figure 5. Two examples of the cumulative probability density functions for the GRB host true flux densities (dashed curves; blue online), based on the luminosityweighted galaxy LFs, as described in Section 3.2. These are then convolved with the observed measurement errors for each field to produce the solid (red) curves which are the predicted CDFs for the measured flux densities. The dotted (green) lines show the formal measured flux density at the location of the GRB in each case. Reading these across on the CDF axes we would expect to find locations drawn randomly from a uniform distribution between 0 and 1 .

(A color version of this figure is available in the online journal.)

formal measured flux density at each GRB position. Note that for GRB 090423 we take a weighted average of the results for both filters, since they straddle $1500 \AA$, while in the analysis of GRB 060927 we use the F110W flux density, being the closest match to $1500 \AA$. If the assumptions we have made are correct, then we would expect these measured flux densities to be drawn randomly and uniformly from 0 to 1 on the cumulative probability axes. We can quantify this by calculating the average value for $\left\langle Y\left(F_{\mathrm{obs}}\right)\right\rangle=0.46$, consistent with an expected value of $0.5 \pm 0.12$ from the Central Limit Theorem.

However, we can also repeat the analysis, but this time fix the LF parameters for all the hosts to be that found at lower redshift. Specifically, we first choose $\alpha=1.73$ and $M^{*}=-20.97$, as measured at $z \approx 3$ by Reddy \& Steidel (2009). Thus, we are testing here whether the hosts could be drawn from an LF whose shape (but not normalization) does not evolve from $z \sim 3$ to high redshift. The results in this case for the same two bursts are shown in Figure 6. Now all the measured flux densities at the GRB locations are close to or below the $Y=0.5$ level, with a mean $\left\langle Y\left(F_{\text {obs }}\right)\right\rangle=0.29$, thus rejecting the model at $\approx 96 \%$ confidence $(\approx 98 \%$ if we took $z=6.5$ for the redshift of
GRB 090429B and averaged the F105W and F160W limits). An alternative test would be to fix the parameters to those found at $z=6-7$ by McLure et al. (2010), $\alpha=1.71$ and $M^{*}=-20.08$. Again, this model is weakly rejected at the $\approx 90 \%$ level $(\approx 94 \%)$.

Our result is not a highly significant, but does support an evolving galaxy LF, with an increasing proportion of star formation occurring in faint galaxies. It also demonstrates that a larger sample and/or deeper limits on host emission can begin to provide important tests of the high- $z$ galaxy LF.

\subsection{Implications for Reionization}

Whether UV radiation from star-forming galaxies is sufficient to bring about and sustain the reionization of the IGM above $z \sim 6$ is a long-standing question (e.g., Loeb 2009). Recently, a number of authors have argued that an increasingly steep faint end to the galaxy LF, motivated by theoretical considerations, may be able to achieve this, without resorting to extremely high Lyman continuum escape fractions (e.g., Bouwens et al. 2012; Lorenzoni et al. 2011; Kuhlen \& Faucher-Giguère 2012). However, the form, steepness, and faint-end cutoff of the galaxy 


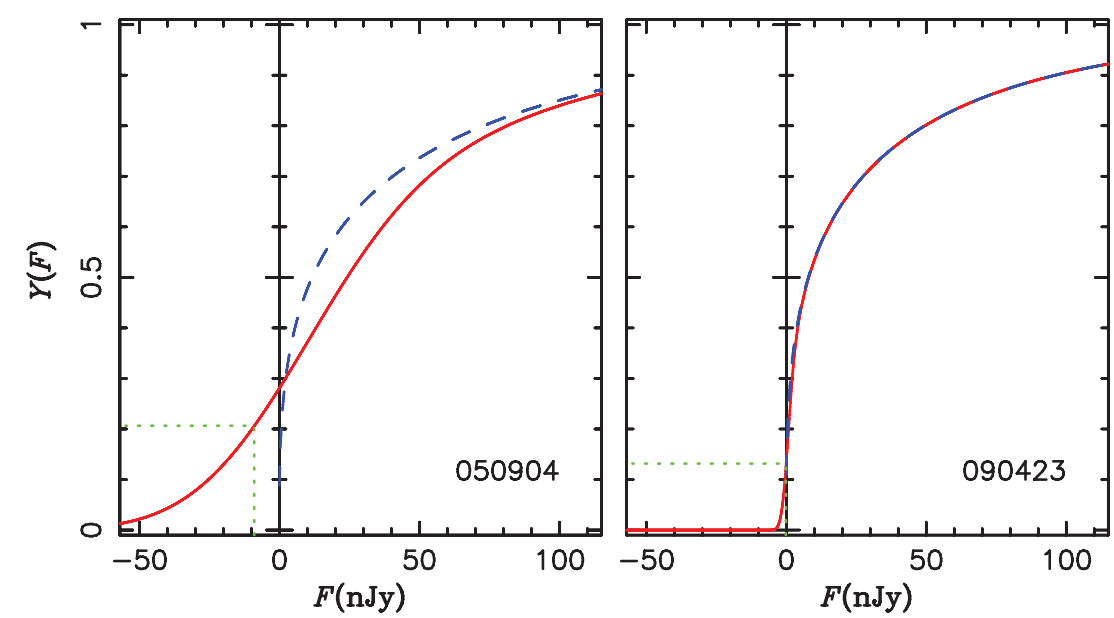

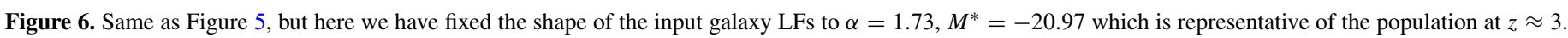
The shallower slope and brighter characteristic luminosity of this LF leads to our faint flux limits corresponding to systematically lower values of the CDF, $Y(F)$. (A color version of this figure is available in the online journal.)

LF at $z>7$ are very poorly constrained by current data, since HST (and even in the longer-term JWST and ground-based 20-40 m class optical/NIR telescopes) can only directly probe the bright end of the LF.

If GRBs are sampling star formation in an unbiased way, as we argue they may be at early times, then they provide an alternative window on the total SFR which would ultimately circumvent the necessity to detect the individual galaxies in which the star formation is occurring. Our results, even from the small sample of high-redshift bursts currently available, already support an evolving galaxy LF over a non-evolving one, and therefore suggest that reionization may be brought about primarily from stars born in very faint protogalaxies.

\subsection{Possible Biases}

It is worth considering further possible physical effects which may be biasing our conclusions. If a significant amount of star formation at high- $z$ is actually dust enshrouded, which we believe is unlikely, then it would impact the observability of GRBs and their hosts, as well as the LBG samples. One would generally expect that the GRBs for which afterglows are detected and redshifts estimated would typically be in the lower dust systems, if there is indeed a wide range, and so in that sense, their hosts could still be compared directly to the (also preferentially dust free) LBG samples. From the point of view of reionization, of course it is the low-dust star formation that is more likely to have a high escape fraction of ionizing radiation, so our conclusions are likely to be valid in that respect.

As discussed in Section 1, we should also be concerned about possible GRB metallicity sensitivity. Both theoretical considerations and observational evidence (the blue colors of the LBG samples) argue that few star-forming galaxies at $z>6$ will have high metallicities (e.g., supersolar) and therefore the low rate of GRBs in such systems seen at low redshift is not likely to be an important factor at high- $z$. Of course, it could be that a contrary effect becomes important at some point, for instance if very low metallicity populations produce fewer and/or fainter GRBs. However, if that were the case then we would expect to lose, if anything, the lower mass halos, where any metals produced are most easily lost. Hence, such an effect would seem unlikely to result in finding an unusually faint population of hosts, and so again our basic conclusion would only be strengthened.

\section{CONCLUSIONS}

We have presented HST observations of a sample of six GRB host galaxies beyond $z \sim 5$. One host, that of GRB 060522 at $z=5.11$ may be marginally detected, but the others are undetected to deep limits, typically $H_{\mathrm{AB}} \sim 28$ at $2 \sigma$. If GRBs are good tracers of the locations of star formation at high- $z$ our results confirm that much, and probably the majority of, star formation then was taking place in small galaxies that are too faint to be detected even in the various HST deep-field surveys. While the sample is small, the joint probability that none of the hosts are detected is consistent with a galaxy LF which is rapidly evolving to higher redshifts, and marginally inconsistent with an LF whose shape does not evolve.

Our analysis does rely on two assumptions: that environmental conditions, such as abundance variations, do not produce appreciable variations in the SFR to GRB-rate ratio from galaxy to galaxy, and that dust content is generally negligible. Both of these are consistent with our current understanding of early galaxies, and of GRBs, but doubtless work is required to further clarify these issues. In terms of the effect of dust, we note that from the point of view of reionization, the uncorrected LF is really what we are interested in, since it is largely the unobscured star formation which will contribute to the intergalactic UV radiation field. However, if there were significant dust in some GRB hosts, it would weaken the connection between GRB-likelihood and apparent UV luminosity which we have assumed.

This work demonstrates the potential power of GRB-selected galaxy samples to quantify the amount of star formation occurring in faint galaxies at early times, which is essential for understanding the budget of UV photons and their role in reionizing the Universe. In the future, deep imaging of larger samples of GRBs at high- $z$, combined with better understanding of any environmental dependencies of GRB production, could provide much more stringent constraints on the faint end of the galaxy LF. Specifically, such a sample could in principle be used to fit for all the LF shape parameters, including the minimum cutoff luminosity, or, more empirically, simply determine the relative SFR fraction in bright (detected) galaxies compared to that in 
faint (undetected) galaxies, without any prior assumption about the form of the LF.

Just as this paper was submitted, two other papers on the same topic appeared as preprints. Basa et al. (2012) have reported deep VLT observations of three high- $z$ fields, of GRBs 060522 , 060927, and 080913. The point-source magnitudes reached are rather shallower than the HST limits, and their non-detections are therefore consistent with our results. Trenti et al. (2012) instead performed a theoretical analysis, aimed at predicting the fraction of GRB hosts expected to be detected in deep HST imaging at different redshifts, under various model assumptions. For example, they predict that $50 \%$ of GRB hosts at $z \sim 5$ should be detected in a survey reaching $M_{\mathrm{AB}} \sim-18$, consistent with our possible detection of the GRB 060522 host. To the same rest-frame limit, they predict fewer than $20 \%$ of hosts should be detected at $z \sim 8$, again, in agreement with our findings.

Traditional blank-field imaging surveys, while potentially finding larger samples of candidate galaxies, are limited to detecting just the relatively brightest examples at the most extreme redshifts. This may be true even in the era of the $J W S T$, especially given the apparent dearth of $z \sim 10$ candidates located so far in the HUDF (Oesch et al. 2012), which could be indicating an even more rapid evolution of the galaxy LF parameters. The approach we and the other studies mentioned above have adopted provides a crucial complementary insight into early galaxy evolution.

We acknowledge support from STFC. Based on observations made with the NASA/ESA Hubble Space Telescope (HST), obtained from the data archive at the Space Telescope Institute. STScI is operated by the association of Universities for Research in Astronomy, Inc. under the NASA contract NAS 5-26555. These observations are associated with HST programs GO-10616 (PI: Berger), GO-10926 (PI: Tanvir), GO-11189 (PI: Tanvir), and GO-11734 (PI: Levan).

J.P.U.F. acknowledges support from the ERC-StG grant EGGS-278202. The Dark Cosmology Centre is funded by the DNRF.

We thank Justin Read for useful discussions.

\section{REFERENCES}

Basa, S., Cuby, J. G., Savaglio, S., et al. 2012, arXiv:1201.6383 Berger, E., Chary, R., Cowie, L. L., et al. 2007, ApJ, 665, 102 Berger, E., Kulkarni, S. R., Bloom, J. S., et al. 2002, ApJ, 581, 981 Bloom, J. S., Perley, D. A., Li, W., et al. 2009, ApJ, 691, 723 Bouwens, R. J., Illingworth, G. D., Labbe, I., et al. 2011a, Nature, 469, 504 Bouwens, R. J., Illingworth, G. D., Oesch, P. A., et al. 2010a, ApJ, 709, L133 Bouwens, R. J., Illingworth, G. D., Oesch, P. A., et al. 2010b, ApJ, 708, L69 Bouwens, R. J., Illingworth, G. D., Oesch, P. A., et al. 2011b, ApJ, 737, 90 Bouwens, R. J., Illingworth, G. D., Oesch, P. A., et al. 2012, ApJ, 752, L5 Bunker, A. J., Wilkins, S., Ellis, R. S., et al. 2010, MNRAS, 409, 855 Castro Cerón, J. M., Michałowski, M. J., Hjorth, J., et al. 2006, ApJ, 653, L85 Cenko, S. B., Berger, E., Djorgovski, S. G., Mahabal, A. A., \& Fox, D. B. 2006, GRB Coordinates Network, 5155, 1

Chary, R., Berger, E., \& Cowie, L. 2007, ApJ, 671, 272

Chen, H.-W., Perley, D. A., Pollack, L. K., et al. 2009, ApJ, 691, 152

Chen, H.-W., Perley, D. A., Wilson, C. D., et al. 2010, ApJ, 723, L218

Christensen, L., Hjorth, J., \& Gorosabel, J. 2004, A\&A, 425, 913

Cucchiara, A., Fox, D. B., \& Berger, E. 2009, GRB Coordinates Network, 9209, 1

Cucchiara, A., Levan, A. J., Fox, D. B., et al. 2011, ApJ, 736, 7

D'Avanzo, P., Piranomonte, S., Magazzu, A., \& Mainella, G. 2006, GRB Coordinates Network, 5151, 1

Faucher-Giguère, C., Lidz, A., Zaldarriaga, M., \& Hernquist, L. 2009, ApJ, 703, 1416

Finkelstein, S. L., Papovich, C., Salmon, B., et al. 2011, arXiv:1110.3785

Fruchter, A. S., Levan, A. J., Strolger, L., et al. 2006, Nature, 441, 463
Fynbo, J. P. U., Gorosabel, J., Smette, A., et al. 2005, ApJ, 633, 317

Fynbo, J. P. U., Jakobsson, P., Prochaska, J. X., et al. 2009, ApJS, 185, 526

Fynbo, J. P. U., Prochaska, J. X., Sommer-Larsen, J., Dessauges-Zavadsky, M., \& Møller, P. 2008, ApJ, 683, 321

Fynbo, J. P. U., Starling, R. L. C., Ledoux, C., et al. 2006, A\&A, 451, L47

Gou, L. J., Mészáros, P., Abel, T., \& Zhang, B. 2004, ApJ, 604, 508

Greiner, J., Krühler, T., Fynbo, J. P. U., et al. 2009, ApJ, 693, 1610

Haislip, J. B., Nysewander, M. C., Reichart, D. E., et al. 2006, Nature, 440, 181

Hashimoto, T., Ohta, K., Aoki, K., et al. 2010, ApJ, 719, 378

Hjorth, J., Møller, P., Gorosabel, J., et al. 2003a, ApJ, 597, 699

Hjorth, J., Sollerman, J., Møller, P., et al. 2003b, Nature, 423, 847

Jakobsson, P., Björnsson, G., Fynbo, J. P. U., et al. 2005, MNRAS, 362, 245

Jakobsson, P., Hjorth, J., Malesani, D., et al. 2012, ApJ, 752, 62

Kawai, N., Kosugi, G., Aoki, K., et al. 2006, Nature, 440, 184

Kocevski, D., West, A. A., \& Modjaz, M. 2009, ApJ, 702, 377

Komatsu, E., Smith, K. M., Dunkley, J., et al. 2011, ApJS, 192, 18

Krühler, T., Schady, P., Greiner, J., et al. 2011, A\&A, 526, A153

Kuhlen, M., \& Faucher-Giguère, C.-A. 2012, MNRAS, 423, 862

Küpcü Yoldaş, A., Greiner, J., Klose, S., Krühler, T., \& Savaglio, S. 2010, A\&A, $515, \mathrm{~L} 2$

Lamb, D. Q., \& Reichart, D. E. 2000, ApJ, 536, 1

Le Floc'h, E., Charmandaris, V., Forrest, W. J., et al. 2006, ApJ, 642, 636

Levesque, E. M., Berger, E., Kewley, L. J., \& Bagley, M. M. 2010a, AJ, 139, 694

Levesque, E. M., Kewley, L. J., Graham, J. F., \& Fruchter, A. S. 2010b, ApJ, 712, L26

Levesque, E. M., Soderberg, A. M., Kewley, L. J., \& Berger, E. 2010c, ApJ, 725,1337

Loeb, A. 2009, J. Cosmol. Astropart. Phys., 03, 22

Lorenzoni, S., Bunker, A. J., Wilkins, S. M., et al. 2011, MNRAS, 414, 1455

Madau, P., Pozzetti, L., \& Dickinson, M. 1998, ApJ, 498, 106

McLure, R. J., Dunlop, J. S., Cirasuolo, M., et al. 2010, MNRAS, 403, 960

McLure, R. J., Dunlop, J. S., de Ravel, L., et al. 2011, MNRAS, 418, 2047

McQuinn, M., Lidz, A., Zaldarriaga, M., Hernquist, L., \& Dutta, S. 2008, MNRAS, 388, 1101

Michałowski, M. J., Hjorth, J., Castro Cerón, J. M., \& Watson, D. 2008, ApJ, 672,817

Miralda-Escude, J. 1998, ApJ, 501, 15

Modjaz, M., Kewley, L., Kirshner, R. P., et al. 2008, AJ, 135, 1136

Mortlock, D. J., Warren, S. J., Venemans, B. P., et al. 2011, Nature, 474, 616

Natarajan, P., Albanna, B., Hjorth, J., et al. 2005, MNRAS, 364, L8

Oesch, P. A., Bouwens, R. J., Carollo, C. M., et al. 2010, ApJ, 709, L21

Oesch, P. A., Bouwens, R. J., Illingworth, G. D., et al. 2012, ApJ, 745, 110

Ono, Y., Ouchi, M., Mobasher, B., et al. 2012, ApJ, 744, 83

Patel, M., Warren, S. J., Mortlock, D. J., \& Fynbo, J. P. U. 2010, A\&A, 512, L3

Perley, D. A., Cenko, S. B., Bloom, J. S., et al. 2009, AJ, 138, 1690

Pian, E., Mazzali, P. A., Masetti, N., et al. 2006, Nature, 442, 1011

Pontzen, A., Deason, A., Governato, F., et al. 2010, MNRAS, 402, 1523

Prochaska, J. X., Chen, H.-W., Bloom, J. S., et al. 2007, ApJS, 168, 231

Racusin, J. L., Karpov, S. V., Sokolowski, M., et al. 2008, Nature, 455, 183

Read, J. I., Pontzen, A. P., \& Viel, M. 2006, MNRAS, 371, 885

Reddy, N. A., \& Steidel, C. C. 2009, ApJ, 692, 778

Robertson, B. E., \& Ellis, R. S. 2012, ApJ, 744, 95

Rossi, A., Greiner, J., Krüher, T., et al. 2008, GRB Coordinates Network, 8218, 1

Ruiz-Velasco, A. E., Swan, H., Troja, E., et al. 2007, ApJ, 669, 1

Salvaterra, R., Della Valle, M., Campana, S., et al. 2009, Nature, 461, 1258

Savaglio, S., Glazebrook, K., \& Le Borgne, D. 2009, ApJ, 691, 182

Savaglio, S., Rau, A., Greiner, J., et al. 2011, MNRAS, 420, 627

Schady, P., Dwelly, T., Page, M. J., et al. 2012, A\&A, 537, A15

Schechter, P. 1976, ApJ, 203, 297

Sirianni, M., Jee, M. J., Benítez, N., et al. 2005, PASP, 117, 1049

Stanek, K. Z., Matheson, T., Garnavich, P. M., et al. 2003, ApJ, 591, L17

Stanway, E. R., Bremer, M. N., Tanvir, N. R., Levan, A. J., \& Davies, L. J. M. 2011, MNRAS, 410, 1496

Svensson, K. M., Levan, A. J., Tanvir, N. R., et al. 2012, MNRAS, 421, 25

Svensson, K. M., Levan, A. J., Tanvir, N. R., Fruchter, A. S., \& Strolger, L. 2010, MNRAS, 405, 57

Tagliaferri, G., Antonelli, L. A., Chincarini, G., et al. 2005, A\&A, 443, L1

Tanvir, N. R., Barnard, V. E., Blain, A. W., et al. 2004, MNRAS, 352, 1073

Tanvir, N. R., Fox, D. B., Levan, A. J., et al. 2009, Nature, 461, 1254

Tanvir, N. R., \& Jakobsson, P. 2007, Phil. Trans. R. Soc., 365, 1377

Thompson, R. I., Storrie-Lombardi, L. J., Weymann, R. J., et al. 1999, AJ, 117, 17 
Thöne, C. C., Kann, D. A., Jóhannesson, G., et al. 2010, A\&A, 523, A70 Totani, T., Kawai, N., Kosugi, G., et al. 2006, PASJ, 58, 485

Trenti, M., Perna, R., Levesque, E. M., Shull, J. M., \& Stocke, J. T. 2012, ApJ, 749, L38

Trenti, M., Stiavelli, M., Bouwens, R. J., et al. 2010, ApJ, 714, L202

Vanzella, E., Pentericci, L., Fontana, A., et al. 2011, ApJ, 730, L35

Vreeswijk, P. M., Ellison, S. L., Ledoux, C., et al. 2004, A\&A, 419, 927

Weisz, D. R., Dolphin, A. E., Dalcanton, J. J., et al. 2011, ApJ, 743, 8
Wijers, R. A. M. J., Bloom, J. S., Bagla, J. S., \& Natarajan, P. 1998, MNRAS, 294, L13

Wolf, C., \& Podsiadlowski, P. 2007, MNRAS, 375, 1049

Woosley, S. E., \& Heger, A. 2006, ApJ, 637, 914

Yoon, S.-C., \& Langer, N. 2005, A\&A, 443, 643

Yüksel, H., Kistler, M. D., Beacom, J. F., \& Hopkins, A. M. 2008, ApJ, 683, L5

Zafar, T., Watson, D., Fynbo, J. P. U., et al. 2011a, A\&A, 532, A143

Zafar, T., Watson, D. J., Tanvir, N. R., et al. 2011b, ApJ, 735, 2 